\title{
De la ingeniería a la idoneidad didáctica en educación matemática
}

\author{
Juan D. Godino
}

\begin{abstract}
Resumen: La Didáctica de la matemática tiene un componente científico (descriptivo, explicativo y predictivo) y un componente tecnológico (prescriptivo), el cual implica el diseño y experimentación de intervenciones educativas óptimas en cada contexto y circunstancias. En consecuencia, se aplican y desarrollan teorías y métodos generales de investigación educativa, así como teorías instruccionales específicas y locales. En este trabajo analizamos tres teorías ampliamente usadas en Didáctica de la matemática destacando los rasgos que incorporan relacionados con el componente tecnológico de la Didáctica, esto es, como teorías de diseño instruccional o ingeniería didáctica. Se trata de la Teoría de Situaciones, el Enfoque Antropológico y la Educación Matemática Realista. Así mismo, describimos la Teoría de la Idoneidad Didáctica, como un componente del Enfoque Ontosemiótico, que aborda el problema axiológico de identificación y estructuración de criterios de optimización de los procesos de instrucción matemática, mediante los cuales se puede salvar la brecha entre la ingeniería didáctica y la práctica de la enseñanza. Finalmente se analizan las concordancias y complementariedades entre las cuatro teorías mencionadas. La clarificación y comparación de los criterios de idoneidad didáctica de diferentes marcos teóricos y su articulación en un sistema coherente supone un programa de investigación que apenas sugerimos en este artículo.
\end{abstract}

Palabras clave: Educación matemática. Ingeniería didáctica. Diseño instruccional. Idoneidad didáctica. Comparación de teorías.

\section{From engineering to didactic suitability in mathematics education}

Juan D. Godino

Doctorado en Matemáticas por la Universidad de Granada. Profesor de la Universidad de Granada, Granada, España. https://orcid.org/0000-0001-8409-0258 jdgodino@gmail.com

Recebido em 06/07/2021 Aceito em 05/09/2021 Publicado em 08/09/2021

Abstract: Didactics of mathematics has a scientific component (descriptive, explanatory and predictive) and a technological component (prescriptive), which involves the design and experimentation of optimal educational interventions in each context and circumstances. Consequently, general educational research theories and methods are applied and developed, as well as specific and local instructional theories. In this paper we analyse three theories widely used in Didactics of Mathematics highlighting the features they incorporate related to the technological component of Didactics, that is, as theories of instructional design or didactic engineering. These are the Theory of Situations, the Anthropological Approach and Realistic Mathematics Education. Likewise, we describe the Theory of Didactic Suitability, as a component of the Onto-semiotic Approach, which addresses the axiological problem of identifying and structuring criteria for optimising mathematical instructional processes, by means of which the gap between didactic engineering and teaching practice can be bridged. Finally, the concordances and complementarities between the four mentioned theories are analysed. The clarification and comparison of the didactic suitability criteria of different theoretical frameworks and their articulation into a coherent system is a research programme that we only suggest in this article.

Keywords: Mathematics education. Teaching and learning. Didactical engineering. Instructional design. Didactical suitability. Comparing theories.

\section{Da engenharia à idoneidade didática no ensino da matemática}

Resumo: A didática da matemática tem um componente científico (descritivo, explicativo e preditivo) e um componente tecnológico (prescritivo), que envolve a concepção e experimentação de intervenções educacionais ótimas em cada contexto e circunstância. Consequentemente, teorias e métodos gerais de pesquisa educacional são aplicados e desenvolvidos, assim como teorias instrucionais específicas e locais. Neste artigo analisamos três teorias amplamente utilizadas na Didática da Matemática destacando as características que elas incorporam relacionadas ao componente tecnológico da Didática, ou seja, como teorias 
de desenho instrucional ou engenharia didática. Estas são a Teoria das Situações, a Abordagem Antropológica e a Educação em Matemática Realista. Da mesma forma, descrevemos a Teoria da Idoneidade Didática, como um componente da Abordagem Ontosemiótica, que enfrenta o problema axiológico de identificar e estruturar critérios para a otimização dos processos de instrução matemática, através do qual a brecha entre a engenharia didática e a prática didática pode ser superada. Finalmente, são analisadas as concordâncias e complementaridades entre as quatro teorias mencionadas. 0 esclarecimento e comparação dos critérios de idoneidade didática de diferentes quadros teóricos e sua articulação em um sistema coerente é um programa de pesquisa que apenas sugerimos neste artigo.

Palavras-chave: Educação matemática. Engenharia didática. Desenho instrucional. Idoneidade didática. Comparação de teorias.

\section{Introducción}

En didáctica de la matemática se ha enfatizado el componente de disciplina científica, y por tanto, el carácter descriptivo, explicativo y predictivo de las investigaciones que se realizan en este campo. Sin embargo, dado que el objeto de estudio son los procesos de enseñanza y aprendizaje en contexto reales, es inevitable el compromiso con la intervención sobre dichos procesos para hacerlos lo más idóneos posibles (GODINO; BATANERO y FONT, 2019). Esto supone reconocer que la didáctica de la matemática tiene también un carácter tecnológicoprescriptivo, y por tanto en su seno se deben desarrollar teorías de diseño educativo. Dichas teorías están dirigidas a la práctica, describen métodos educativos y las situaciones en las que dichos métodos deberían utilizarse. "Una teoría de diseño educativo es una teoría que ofrece una guía explícita sobre la mejor forma de ayudar a que la gente aprenda y se desarrolle. Los tipos de conocimientos y desarrollo pueden ser cognitivos, emocionales, físicos y espirituales" (REIGELUTH, 2000, p. 15).

En este trabajo se aborda la problemática del diseño instruccional en educación matemática desde el punto de vista de enfoques teóricos internos a la Didáctica de las Matemáticas, en particular de la Teoría de Situaciones Didácticas (TSD) (BROUSSEAU, 1986; 1997), la Teoría Antropológica de lo Didáctico (TAD) (CHEVALLARD, 1992; 1999), la Educación Matemática Realista (EMR) (FREUDENTHAL, 1973; 1983; VAN DEN HEUVEL-PANHUIZEN y DRIJVERS, 2014), y el Enfoque Ontosemiótico del conocimiento y la instrucción matemáticos (EOS) (GODINO, 2002; GODINO; BATANERO y FONT, 2007).

Para intentar salvar la brecha entre la investigación teórica y los problemas de la práctica docente se han desarrollado las llamadas "investigaciones basadas en el diseño" (IBD) (BROWN, 1992; KELLY; LESH y BAEK, 2008), las cuales constituyen una familia de aproximaciones metodológicas orientadas al estudio del aprendizaje en contexto. Utiliza el diseño instruccional y la investigación sistemática de estrategias y herramientas instruccionales, tratando que sean 
interdependientes, sobreentendiéndose que la investigación incluye no solo la fase de diseño, sino también la experimentación en contextos de clase y la evaluación de sus resultados. En educación matemática, las IBD se realizan aplicando diferentes teorías de base en los diseños e interpretación de los resultados. Así, Artigue (2015) describe la metodología de la ingeniería didáctica como un tipo de IBD, basada en la TSD, en la TAD y en otras teorías. La Ingeniería Didáctica (ID) (ARTIGUE, 1989; 2011) se presenta usualmente con una doble faceta: como "metodología de investigación", y como conjunto de medios o recursos para la enseñanza de temas específicos, elaborados teniendo en cuenta los resultados de la investigación. "La ingeniería didáctica surgió, así, como una metodología de investigación y desarrollo basada en realizaciones en el aula en forma de secuencias de lecciones, informadas por la teoría y poniendo a prueba las ideas teóricas" (ARTIGUE, 2015, p. 469). También Chevallard (2009) distingue entre ingeniería didáctica de investigación e ingeniería didáctica de desarrollo, entre las cuales existe una tensión al estar la primera orientada a la producción de nuevos conocimientos y la segunda al uso práctico de los mismos.

Godino, Batanero, Contreras, Estepa, Lacasta y Wilhelmi (2013) analizan las características de la IBD y la ID y concluyen que, dado que la ID se puede basar en distintos marcos teóricos, la ID y el diseño instruccional describen el mismo tipo de investigaciones didácticas. Como sugieren Bakker y van Eerde (2015), estas investigaciones de diseño se pueden implementar aplicando la EMR como acción útil en el proceso de diseño, complementada con otras teorías locales, específicas del contenido matemático abordado.

Las investigaciones de diseño instruccional o de ingeniería didáctica, cualquiera que sea la teoría de base en la cual se apoyen, responden a cuestiones del tipo ¿Qué resultados en términos de aprendizaje se obtienen si se realiza una intervención educativa específica en un contexto dado?, esto es, corresponden al esquema, Si $X$ entonces $Y$. Puesto que se realizan en contextos educativos reales, tienen en cuenta la riqueza y complejidad de factores que condicionan la práctica docente. En consecuencia, los recursos elaborados y los conocimientos obtenidos de estas investigaciones tienen el potencial de dar solución a problemas de dicha práctica, los cuales se refieren usualmente a qué matemáticas enseñar y cómo. Sin embargo, dado que el conocimiento que aporta este tipo de investigación es predictivo, no se pueden derivar de ella valoraciones y normas de acción, como se requiere en la intervención eficiente sobre la práctica. La superación de la brecha existente entre el conocimiento científico y la práctica de la enseñanza precisa del desarrollo de teorías que expliciten el sistema de principios axiológicos, así como los criterios valorativos y normativos sobre la acción educativa eficiente, derivados de la investigación 
teórica y aplicada. En el marco del EOS esta interfaz viene dada por la Teoría de la Idoneidad Didáctica (TID) que describimos en la sección 3. Previamente, en la sección 2 describimos las principales características de la TSD, la TAD y la EMR en los aspectos relacionados con el diseño instruccional. En la sección 4 identificamos concordancias y complementariedades de estas teorías con la TID. Concluimos el artículo con una síntesis y algunas observaciones finales.

\section{Teorías de diseño instruccional en didáctica de las matemáticas}

El carácter central del conocimiento matemático, como polo de entrada para el análisis didáctico, es un rasgo clave de la TSD y su desarrollo en la "teoría de los momentos didácticos" (CHEVALLARD, 1997), cuyas principales características resumiremos en las secciones 2.1 y 2.2. Gascón (1998) presenta la TSD como el comienzo de un nuevo enfoque de la didáctica, que pone en un segundo plano los factores de índole cognitiva y la didáctica fundamental de las matemáticas, e interpreta la TAD como uno de los desarrollos naturales de la didáctica fundamental.

De ambas teorías se derivan implicaciones para la acción efectiva sobre la enseñanza y aprendizaje de las matemáticas, aunque su objetivo esencial sea la generación de conocimientos para comprender los procesos de estudio de las matemáticas y los factores que los condicionan. Por ejemplo, si aceptamos postulados antropológicos sobre la actividad matemática se debe atribuir un papel central a las situaciones - problemas como razón de ser de los conocimientos matemáticos; en consecuencia, se puede formular una norma instruccional: para que un proceso de instrucción matemática sea óptimo desde el punto de vista matemático el proceso debe estar basado en el estudio de una secuencia de problemas interconectados representativos del campo correspondiente.

\subsection{La teoría de situaciones didácticas}

En la base de la TSD está la hipótesis epistemológica de que "el conocimiento existe y tiene sentido para el sujeto cognoscente, solo porque representa una solución óptima en un sistema de restricciones" (BROUSSEAU, 1986, p. 368). El acto de conocer está situado en un sistema de restricciones que señalan al sujeto el coste de sus ensayos y errores, mediante el "feedback" sobre sus acciones. El aprendizaje, es entendido como un cambio en las relaciones del sujeto al medio y ocurre cuando la aplicación de nociones previamente construidas es demasiado costosa, y el sujeto está obligado a hacer adaptaciones o incluso rechazos de las mismas. 
La TSD propone un programa de investigación completo para la didáctica de la matemática, que implica estudios epistemológicos, diseño de situaciones didácticas, experimentación, comparación del diseño con los procesos que tienen lugar, revisión de los estudios epistemológicos y del diseño, y estudio de las condiciones de la reproductibilidad de las situaciones. Los aspectos metodológicos de este programa son descritos mediante la ingeniería didáctica. "Vista como una práctica de investigación o desarrollo, la ingeniería didáctica es claramente una práctica del tipo de intervención controlada, y esta intervención está basada en la teoría. En este caso, la teoría es la TDS y esto afecta profundamente la visión del diseño" (ARTIGUE, 2009, p. 10).

En la TSD la génesis artificial de un concepto matemático tiene lugar como resultado de una secuencia de los siguientes tipos de situaciones o estados de un contrato didáctico:

- Situaciones centradas sobre la acción, donde los estudiantes hacen sus primeros intentos por resolver un problema propuesto por el profesor.

- Situaciones enfocadas en la comunicación, donde los estudiantes comunican los resultados de su trabajo a otros estudiantes y al profesor.

- Situaciones centradas sobre la validación, donde se deben usar argumentaciones teóricas más bien que empíricas.

- Situaciones de institucionalización, donde los resultados de las negociaciones y convenciones de las fases previas son resumidas, y la atención se centra sobre los hechos 'importantes', los procedimientos, las ideas, y la terminología 'oficial'.

A partir de la fase de institucionalización, el significado de los términos ya no es un objeto de negociación, sino de corrección, en referencia a las definiciones, las notaciones, los teoremas, los procedimientos aceptados. Dentro de cada una de estas situaciones, hay un componente adidáctico, esto es, un espacio y tiempo donde la gestión de la situación recae enteramente de parte de los estudiantes.

El programa de investigación esbozado por la teoría de situaciones está dirigido a la elaboración de un cierto número de situaciones fundamentales relacionadas con los conceptos matemáticos básicos enseñados en la escuela, que garanticen, en cierto modo su adquisición por los estudiantes cualquiera que fuese el profesor. Ello se debe a que la hipótesis básica de la TSD es que el conocimiento construido o usado en una situación es definido por las restricciones de esta situación, y que, por tanto, creando ciertas restricciones artificiales el profesor es capaz de provocar que los estudiantes construyan un cierto tipo de conocimiento. Esta hipótesis está 
ciertamente más próxima al constructivismo que a las aproximaciones que se derivan de la noción Vygostskiana de zona de desarrollo próximo (SIERPINSKA y LERMAN, 1996).

Desde nuestro punto de vista, la TSD, y la metodología de investigación descrita como ingeniería didáctica, no son concebidas en términos de una "teoría instruccional", sino que constituyen básicamente una epistemología experimental para la didáctica de la matemática. Así mismo, incorporan o asumen una teoría constructivista - piagetiana para el aprendizaje matemático y un enfoque positivista - experimental para la didáctica de las matemáticas, cuyo objetivo debe ser descubrir fenómenos didácticos, y construir situaciones de enseñanza que necesariamente produzcan los aprendizajes pretendidos.

Sin embargo, aunque el objetivo de la TSD no haya sido construir una teoría de la instrucción matemática, de hecho incorpora elementos en dicha dirección: los tipos de situaciones didácticas (acción, formulación, validación, institucionalización), las normas interaccionistas del contrato didáctico, la construcción de situaciones didácticas basadas en problemas matemáticos específicos de cada conocimiento pretendido, etc., sientan las bases para una "ciencia de diseño" para la educación matemática.

\subsection{La teoría de los momentos didácticos. Recorridos de estudio e indagación}

La Teoría Antropológica en didáctica de las matemáticas (TAD) que Chevallard y colaboradores vienen desarrollando (CHEVALLARD, 1992; 1997; 1999) aporta elementos básicos de una epistemología de las matemáticas que amplían y profundizan la teoría del conocimiento que sirve de base a la TSD. Las nociones de praxeología matemática, relación institucional y personal al objeto constituyen extensiones útiles de las nociones de conocimiento y saber de la TSD. Al mismo tiempo la Teoría de los momentos didácticos, complementada con la noción de REI (Recorrido de estudio e investigación), amplia y matiza los tipos de situaciones adidácticas, aportando criterios para el diseño y gestión de los procesos instruccionales. Ambos modelos teóricos comparten la visión de la matemática como actividad humana, orientada hacia la resolución de cierto tipo de tareas o cuestiones problemáticas.

En la TAD, hacer matemáticas consiste en activar una organización matemática, es decir, resolver determinados tipos de problemas con determinados tipos de técnicas (el saber hacer), de manera inteligible, justificada y razonada (mediante el correspondiente saber). El objetivo de un proceso de enseñanza-aprendizaje puede formularse en términos de los componentes de las organizaciones matemáticas que se quieren reconstruir: qué tipos de problemas hay que ser capaz 
de resolver, con qué tipos de técnicas, sobre la base de qué elementos descriptivos y justificativos, en qué marco teórico, etc.

La teoría antropológica propone un modelo del proceso de estudio de las matemáticas en términos de momentos didácticos (CHEVALLARD; BOSCH y GASCÓN, 1997), que puede constituir el esbozo de una teoría de diseño instruccional. Desde nuestro punto de vista, esta teoría es un refinamiento y ampliación del componente instruccional de la teoría de situaciones didácticas. Los tipos de momentos didácticos que se consideran esenciales en el proceso de estudio de una organización matemática son los siguientes: el momento del primer encuentro, exploratorio, del trabajo de la técnica, tecnológico-teórico, institucionalización y evaluación.

El componente de diseño instruccional de la TAD se ha visto reforzado con la introducción de la noción de recorrido de estudio e investigación (REI) y el cambio de paradigma educativo que conlleva (CHEVALLARD, 2009). Se trata de situar como punto de partida de la acción didáctica el "cuestionamiento del mundo", esto es, se parte de cuestiones (situaciones - problemas) centrales para la matemática o pluridisciplinares, en lugar de comenzar por los saberes, considerados como "obras o monumentos" que "se visitan". En lugar de que los alumnos encuentren las obras matemáticas del programa a través de una multiplicidad de actividades de estudio e indagación, cada una de las cuales parte de una cuestión diferente y moviliza obras "auxiliares" diferentes, se investiga la manera de lograr un fuerte grado de integración, derivando todo un conjunto de cuestiones $Q_{i}$ a partir de una cuestión "generatriz" $Q^{*}$. "De esta manera la cuestión $Q^{*}$ requiere una indagación, la cual se concreta en un cierto recorrido de estudio e investigación". (CHEVALLARD, 2009, p. 26). Una cuestión clave es la generatividad de la cuestión $Q^{*}$ de partida que deberá permitir generar cuestiones derivadas que amplían el rango de las praxeologías que pueden intervenir, y por tanto, se pueden estudiar.

El diseño de los REI debe ser tal que, (1) tengan una orientación matemática amplia y no estén centrados en un concepto o tópico aislado y específico; (2) el programa de un curso se puede estudiar a través de un número finito de "grandes cuestiones": un REI aparece como un verdadero "recorrido de descubrimiento", como un "programa de estudio y de investigación".

En cierto modo la TAD propone un cambio de orientación en la búsqueda de situaciones fundamentales, ya que éstas están centradas en un contenido al que hay que atribuir significado mediante situaciones matemáticas apropiadas. La TAD resalta las cuestiones, frecuentemente codisciplinares, en cuya resolución intervienen sistemas praxeológicos diversos. Aunque comparte con la TSD la pedagogía de la reinvención hay un desplazamiento del centro: ahora no es el objeto conceptual el punto de partida, sino los campos de problemas. Se propone un cambio de 
paradigma del cuestionamiento del mundo y la pedagogía basada en la indagación frente al paradigma del inventario de saberes y la transmisión.

Otras dos nociones de la TAD con implicaciones para el diseño instruccional son, (1) La escala de niveles de codeterminación, que puede ayudar a que los diseños sean sensibles a los diferentes tipos de restricciones a que la acción didáctica está sometida, desde el nivel de civilización al nivel del tema matemático específico abordado; (2) La dialéctica de los media y los milieu que aparece a priori como una herramienta potente para abordar el modo en que la evolución tecnológica cambia radicalmente el acceso a la información, y las características de los milieu con los que podemos interactuar para desarrollar el conocimiento.

\subsection{Educación matemática realista}

La EMR se presenta directamente por destacados investigadores que la aplican y desarrollan (VAN DEN HEUVEL-PANHUIZEN y DRIJVERS, 2014) como una teoría de la instrucción específica para el dominio de las matemáticas, siendo uno de sus rasgos característicos el dar una posición prominente en el proceso de aprendizaje al uso de situaciones "realistas". "Estas situaciones sirven como fuente para iniciar el desarrollo de conceptos, herramientas y procedimientos matemáticos y como contexto en el que los alumnos pueden, en una etapa posterior, aplicar sus conocimientos matemáticos, que luego se hacen gradualmente más formales y generales y menos específicos del contexto". (p. 521).

La EMR está basada en gran medida en las reflexiones y propuestas de Freudenthal (1973; 1983; 1991) sobre las matemáticas y su aprendizaje. Este autor consideró las matemáticas como una actividad humana, que, por tanto, deberían ser aprendidas no como un sistema cerrado, sino como una actividad de matematización de la realidad. La EMR asumen seis principios básicos para la enseñanza de las matemáticas (VAN DEN HEUVEL-PANHUIZEN y DRIJVERS, 2014):

\section{Principio de actividad}

El principio de actividad significa que en el RME los alumnos son tratados como participantes activos en el proceso de aprendizaje. También hace hincapié en que las matemáticas se aprenden mejor haciendo matemáticas, lo que se refleja fuertemente en la interpretación de Freudenthal de las matemáticas como actividad humana, así como en la idea de matematización de Freudenthal (1991). 


\section{Principio de realidad}

El principio de realidad puede reconocerse en el RME de dos maneras. En primer lugar, expresa la importancia que se concede al objetivo de la educación matemática, que incluye la capacidad de los alumnos para aplicar las matemáticas en la resolución de problemas de la "vida real". En segundo lugar, significa que la educación matemática debe partir de situaciones problemáticas que sean significativas para los alumnos, lo que les ofrece la oportunidad de atribuir un significado a las construcciones matemáticas que desarrollan mientras resuelven los problemas. En lugar de comenzar con la enseñanza de abstracciones o definiciones que se aplicarán más tarde, en el RME la enseñanza comienza con problemas en contextos ricos que requieren una organización matemática 0 , en otras palabras, que pueden ser matematizados y ponen a los alumnos en la pista de estrategias de solución informales relacionadas con el contexto como primer paso en el proceso de aprendizaje.

\section{Principio de nivel}

El principio de nivel subraya que el aprendizaje de las matemáticas implica que los alumnos pasen por varios niveles de comprensión: desde las soluciones informales relacionadas con el contexto, pasando por la creación de varios niveles de atajos y esquematizaciones, hasta la adquisición de conocimientos sobre cómo se relacionan los conceptos y las estrategias. Los modelos son importantes para tender un puente entre las matemáticas informales relacionadas con el contexto y las matemáticas más formales. Para cumplir esta función de puente, los modelos tienen que pasar de ser un "modelo de" una situación particular a un "modelo para" todo tipo de situaciones diferentes, pero equivalentes (VAN DEN HEUVEL-PANHUIZEN, 2003). En particular, para la enseñanza de las operaciones con números, este principio de nivel se refleja en el método didáctico de "esquematización progresiva", tal y como fue sugerido por Treffers (1987) y en el que los métodos transparentes de cálculo con números enteros evolucionan gradualmente hacia algoritmos basados en dígitos.

\section{Principio de entrelazamiento}

El principio de entrelazamiento significa que los dominios de contenido matemático, como el número, la geometría, la medida y el tratamiento de datos, no se consideran capítulos aislados del plan de estudios, sino que están fuertemente integrados. A los alumnos se les ofrecen problemas ricos en los que pueden utilizar diversas herramientas y conocimientos matemáticos. Este principio también se aplica dentro de los dominios. Por ejemplo, dentro del dominio del sentido 
numérico, la aritmética mental, la estimación y los algoritmos se enseñan en estrecha conexión entre sí.

\section{Principio de interactividad}

El principio de interactividad del RME significa que el aprendizaje de las matemáticas no es sólo una actividad individual, sino también una actividad social. Por lo tanto, el RME favorece los debates en toda la clase y el trabajo en grupo, que ofrecen a los estudiantes la oportunidad de compartir sus estrategias e invenciones con los demás. De este modo, los estudiantes pueden obtener ideas para mejorar sus estrategias. Además, la interacción suscita la reflexión, lo que permite a los estudiantes alcanzar un mayor nivel de comprensión.

\section{Principio de orientación}

El principio de orientación se refiere a la idea de Freudenthal de "reinvención guiada" de las matemáticas. Implica que en la RME los profesores deben tener un papel proactivo en el aprendizaje de los alumnos y que los programas educativos deben contener escenarios que tengan el potencial de funcionar como palanca para alcanzar cambios en la comprensión de los alumnos. Para ello, la enseñanza y los programas deben basarse en trayectorias de enseñanzaaprendizaje coherentes a largo plazo.

A partir de estos principios generales de enseñanza, se han desarrollado a lo largo del tiempo una serie de teorías locales de instrucción y secuencias didácticas paradigmáticas centradas en temas matemáticos específicos. La base para llegar a estas teorías de instrucción locales se realiza mediante la investigación de diseño, que implica un proceso cíclico de experiencias guiadas por la teoría, incluyendo el diseño de secuencias de enseñanza y su puesta a prueba en experimentos de enseñanza, seguido de un análisis retrospectivo que puede conducir a los ajustes necesarios del diseño (COBB y GRAVEMEIJER, 2008).

\section{Teoría de la idoneidad didáctica basada en el EOS}

EI EOS ha surgido con el propósito de articular diferentes puntos de vista y nociones teóricas sobre el conocimiento matemático, su enseñanza y aprendizaje (GODINO et al., 2007). Se adopta una aproximación al estudio de los problemas didácticos desde un paradigma de complejidad sistémica, como el que propone Morin (1994), con el que es posible encontrar complementariedades por encima de las divergencias aparentes. Se reconoce la complejidad ontosemiótica de las prácticas matemáticas y didácticas, los factores ecológicos que las 
determinan y las interacciones entre las facetas epistémica, cognitiva, afectiva, interaccional, mediacional y ecológica que intervienen en los procesos de estudio de las matemáticas.

El EOS aborda el problema tecnológico de optimización del proceso de instrucción mediante investigaciones del tipo ingeniería o diseño didáctico (GODINO et al., 2014). Los resultados de estas investigaciones, interpretados desde el marco teórico del EOS, tratan de dar respuestas tecnológicas a la problemática docente sobre "qué enseñar y cómo" en circunstancias locales. No obstante, dichas investigaciones se basan en las respuestas a los problemas previos de tipo descriptivo y explicativo implicados, los cuales son propios de la investigación de tipo fundamental orientada a la comprensión de los fenómenos (Figura 1). Como se indica en esta figura, entre los problemas predictivos propios de las investigaciones de diseño y el problema de la práctica docente, usualmente abordado como indagación profesional, hay una separación que debe ser salvada mediante la elaboración de investigaciones del tipo meta-análisis que indague sobre los principios, valores y medios asumidos por la investigación básica y aplicada sobre los cuales haya un cierto grado de consenso para ser aplicados en la práctica educativa. Dado que los fines educativos y los currículos pueden ser diversos, se abre aquí un área problemática de indagación que describimos en la Figura 1 como problemas valorativos, para los cuales se viene desarrollando en el EOS la Teoría de la Idoneidad Didáctica (TID). En esta teoría se explicitan y estructuran los principios axiológicos y criterios de optimización de los procesos de enseñanza y aprendizaje que están implícitamente asumidos en la comunidad de investigación, incorporando algunos propios derivados de los supuestos ontosemióticos sobre el conocimiento y la instrucción matemáticos del EOS.

Figura 1. Problemas y tipos de investigaciones educativas

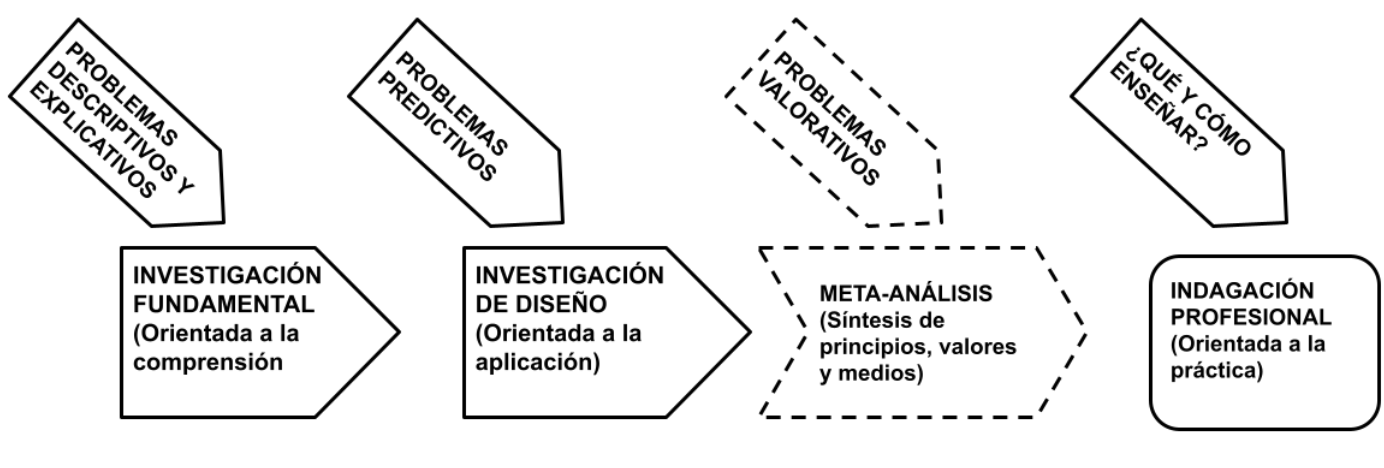

Fuente: Godino et al. (2021, p.13)

La noción de idoneidad didáctica ha sido introducida dentro del EOS como herramienta de paso desde una didáctica descriptiva - explicativa a una didáctica normativa, esto es, orientada hacia la intervención efectiva en el aula. Se asume, por tanto, que la didáctica de la matemática, 
tiene tanto un componente científico como tecnológico (GODINO et al., 2019). Por ello puede servir de punto de partida para una teoría de diseño instruccional que tenga en cuenta, de manera sistémica, las dimensiones epistémica, cognitiva, afectiva, interaccional, mediacional y ecológica implicadas en los procesos de instrucción de las áreas curriculares específicas.

En el EOS se entiende la idoneidad didáctica de un proceso de enseñanza-aprendizaje como el grado en que éste (o una parte del mismo) reúne ciertas características que permiten calificarlo como óptimo o adecuado para conseguir la adaptación entre los significados personales logrados por los estudiantes (aprendizaje) y los significados institucionales pretendidos 0 implementados (enseñanza), teniendo en cuenta las circunstancias y recursos disponibles (entorno). La idoneidad didáctica es un rasgo graduable que supone la articulación coherente de las siguientes seis facetas (GODINO et al., 2007):

- Idoneidad epistémica: grado de representatividad de los significados institucionales implementados (o pretendidos), respecto de un significado de referencia.

- Idoneidad cognitiva: grado en que significados implementados están en la zona de desarrollo potencial de los alumnos, así como la proximidad de los significados personales logrados a los significados implementados.

- Idoneidad interaccional: grado en que las configuraciones y trayectorias didácticas permiten identificar conflictos semióticos potenciales (que se puedan detectar a priori), y resolver los conflictos que se producen durante el proceso de instrucción.

- Idoneidad mediacional: grado de disponibilidad y adecuación de los recursos materiales y temporales necesarios para el desarrollo del proceso de enseñanza-aprendizaje.

- Idoneidad afectiva: grado de implicación (interés, motivación,...) del alumnado en el proceso de instrucción.

- Idoneidad ecológica: grado en que el proceso de instrucción se ajusta al proyecto educativo del centro, la escuela y la sociedad y a los condicionamientos del entorno en que se desarrolla.

Partimos de la base de que en las ciencias sociales y educativas es posible formular criterios de idoneidad, en la forma de juicios de valor, "Se debería hacer esto y no aquello", en aquellas circunstancias en que dichos juicios de valor tienen carácter social y es posible explicitar un fundamento para su formulación. Dichos juicios conllevan una racionalidad, por lo que pueden ser objeto de escrutinio científico (BUNGE, 1999; LACEY, 1999; RUGINA, 1998). 
En la Figura 2 se incluye el sistema de componentes a tener en cuenta en cada una de las facetas de la idoneidad didáctica. Este esquema desarrolla y organiza el modelo incluido en Godino (2013) donde se propone un sistema de indicadores que pueden guiar al docente en el diseño, implementación y evaluación de procesos de enseñanza y aprendizaje de las matemáticas con alta idoneidad didáctica. En la Tabla 1 incluimos la formulación de criterios de idoneidad para cada una de las seis facetas. En Godino et al. (2021) se formulan criterios específicos para los componentes de las facetas epistémica y cognitiva.

En un proceso de instrucción específico hay que tener en cuenta las circunstancias contextuales y personales de los sujetos implicados, por lo que es inevitable centrar la atención en una parte del significado global. Además, dado que la matemática no es solo una trama de objetos histórica y culturalmente construidos, sino también una actividad de las personas, la instrucción debe tener en cuenta los procesos matemáticos implicados (problematización, representación, definición, generalización, modelización, ...). En consecuencia, asumiendo como principio axiológico el proponer o implementar unas "buenas matemáticas", se sigue que los significados seleccionados deben ser representativos del significado global y ofrecer riqueza de procesos en su desarrollo (BREDA: FONT y PINO-FAN, 2018).

Figura 2. Facetas y componentes de la idoneidad didáctica

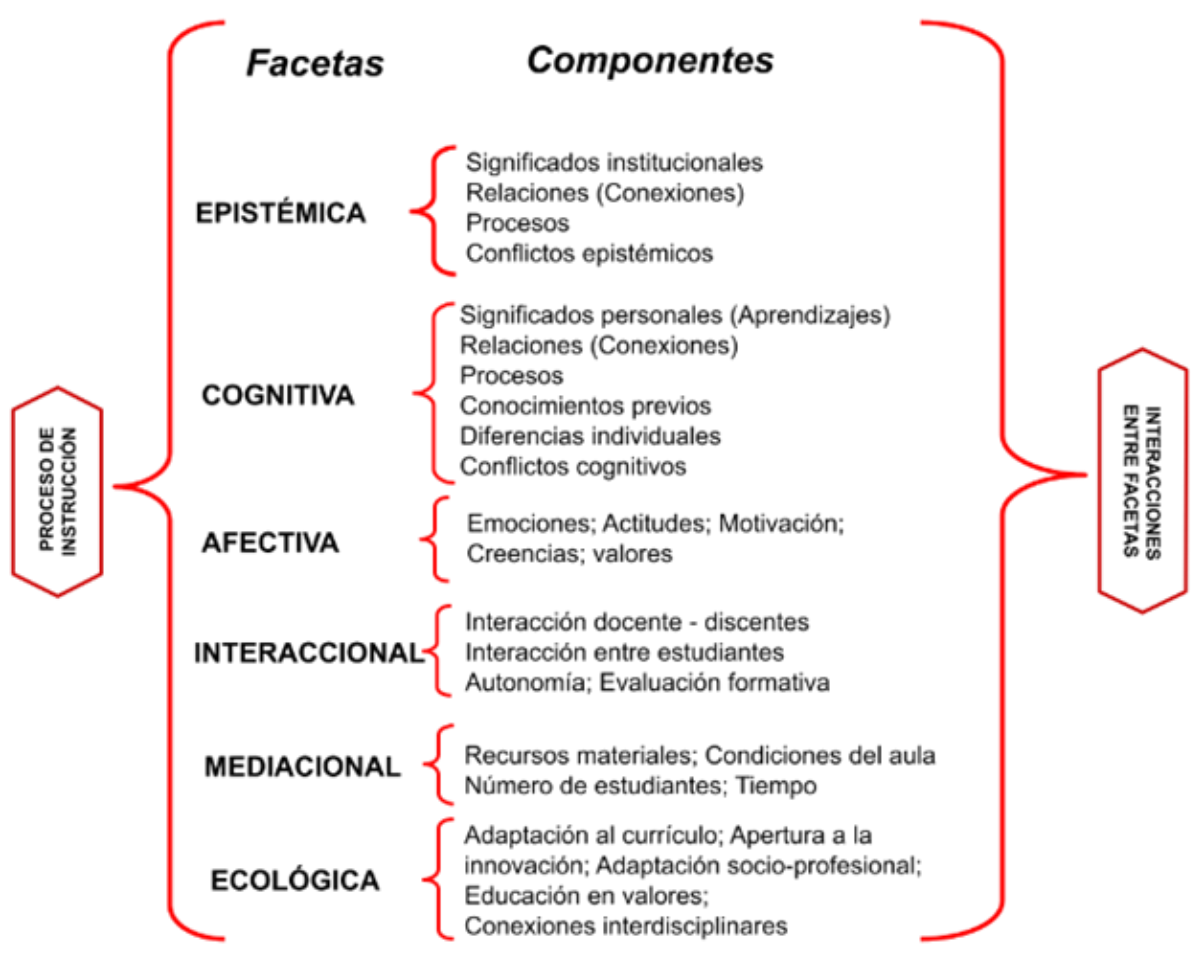

Fuente: Godino et al. (2021, p. 10) 
El criterio o norma general de idoneidad cognitiva está inspirado en el concepto de zona de desarrollo potencial (VYGOTSKY, 1934), por lo que los objetivos de aprendizaje deben implicar el desarrollo de conocimientos y competencias matemáticas valiosas que impliquen un esfuerzo alcanzable para el estudiante, con el apoyo del profesor, los compañeros, los conocimientos previos y capacidades individuales (principio de equidad). Se asume un aprendizaje relacional y con comprensión de los significados institucionales. El estudiante no es un mero sujeto cognitivo que se apropia de unos contenidos y competencias matemáticas sino una persona, con intereses, necesidades y aspiraciones propias, que son contempladas en la faceta afectiva.

Respecto al criterio de idoneidad interaccional se considera necesario tener en cuenta la complejidad ontosemiótica del conocimiento matemático y su naturaleza sociocultural. En consecuencia, se deberá implementar un modelo didáctico basado en la colaboración entre el profesor y los estudiantes, que supere el dilema entre la transmisión del conocimiento y la indagación por los propios estudiantes. Al tener en cuenta la complejidad ontosemiótica del conocimiento matemático el modelo didáctico que se propone desde el EOS requiere tener en cuenta una triple dialéctica entre profesor-contenido, profesor-estudiantes y estudiantescontenido, superando el dilema objetivismo-constructivismo en educación matemática (GODINO y BURGOS, 2020).

Tabla 1. Criterios de idoneidad generales para las seis facetas

\begin{tabular}{|l|l|}
\hline Facetas & Criterios \\
\hline Epistémica & $\begin{array}{l}\text { Representatividad } \\
\text { Los significados institucionales del contenido y las configuraciones de objetos y } \\
\text { procesos implementados deberían ser representativos del significado global de } \\
\text { referencia, teniendo en cuenta las circunstancias contextuales y personales de } \\
\text { los sujetos implicados. }\end{array}$ \\
\hline Cognitiva & $\begin{array}{l}\text { Proximidad y reto alcanzable } \\
\text { Los objetivos de aprendizaje deberían suponer un reto cognitivo alcanzable } \\
\text { para los estudiantes, teniendo en cuenta las circunstancias contextuales y } \\
\text { personales de los sujetos implicados. }\end{array}$ \\
\hline Afectiva & $\begin{array}{l}\text { Implicación } \\
\text { El proceso de instrucción debería lograr el mayor grado posible de implicación del } \\
\text { alumnado (interés, motivación, autoestima, disposición,... ). }\end{array}$ \\
\hline Interaccional & $\begin{array}{l}\text { Negociación } \\
\text { Las configuraciones y trayectorias didácticas que se implementen deberían } \\
\text { permitir identificar los conflictos semióticos potenciales y poner los medios } \\
\text { adecuados para su resolución. }\end{array}$ \\
\hline Mediacional & $\begin{array}{l}\text { Disponibilidad } \\
\text { Se debería disponer de los recursos materiales y temporales adecuados para el } \\
\text { desarrollo óptimo del proceso de enseñanza y aprendizaje. }\end{array}$ \\
\hline
\end{tabular}




\begin{tabular}{|l|l|}
\hline Ecológica & $\begin{array}{l}\text { Adaptación } \\
\text { El proceso de instrucción debería estar en concordancia con el proyecto } \\
\text { educativo del centro y la sociedad, teniendo en cuenta los condicionamientos del } \\
\text { entorno en que se desarrolla y las innovaciones basadas en la investigación } \\
\text { educativa. }\end{array}$ \\
\hline
\end{tabular}

Fuente: Elaboración propia.

El sistema de criterios e indicadores de idoneidad para las distintas facetas y componentes que intervienen en los procesos de instrucción es un instrumento que permite valorar e identificar puntos de mejora para cada fase de un diseño instruccional, y por tanto se convierte en un instrumento de apoyo para la reflexión sobre la propia práctica educativa de los profesores (investigación-acción), así como un instrumento de apoyo en la fase de análisis retrospectivo de las investigaciones basadas en el diseño.

\section{Interpretación de la TSD, TAD y EMR desde la perspectiva de la idoneidad didáctica}

En este apartado interpretamos como criterios de idoneidad algunos principios didácticos de la TSD, la TAD y la EMR, aplicando las facetas y componentes de la idoneidad didáctica que propone el EOS. Esto permitirá identificar algunas concordancias y complementariedades entre estos marcos teóricos. Debemos reconocer, no obstante, que el análisis aquí realizado es muy limitado, dada su complejidad; su ampliación y profundización deberá ser objeto de otros trabajos.

\subsection{Faceta epistémica}

Las cuatro teorías concuerdan en atribuir un papel central a las situaciones-problemas (cuestionestareas) para el logro de una alta idoneidad epistémica en el diseño de procesos instruccionales. La caracterización de situaciones fundamentales para los distintos saberes incluidos en el currículo matemático escolar es uno de sus objetivos prioritarios en la TSD. La noción de recorrido de estudio e investigación de la TAD fija la atención en la búsqueda de cuestiones generativas de las praxeologías matemáticas que constituyen la finalidad de un proyecto educativo.

Así mismo, los principios de actividad y de realidad de la EMR apoyan la consideración de los indicadores recogidos en la Tabla 1 como indicadores de idoneidad epistémica. Para Freudenthal (1991) las matemáticas son una actividad humana. "No hay matemáticas sin matematización", actividad que puede ser de aplicación para resolver problemas del entorno, o problemas de reorganización del propio conocimiento matemático. La EMR propone como heurística para el diseño de situaciones que den sentido a los objetos matemáticos (conceptos, procedimientos, etc.) la fenomenología didáctica, consistente en la búsqueda en la historia y epistemología de las matemáticas de los tipos de fenómenos de la vida real o internos a la propia matemática que son 
organizados por tales objetos, considerados por Freudhental como objetos mentales. "Partiendo de la base de que las matemáticas han surgido como resultado de la resolución de problemas prácticos, podemos suponer que las aplicaciones actuales abarcan los fenómenos que originalmente había que organizar" (GRAVEMEIJER, 2020, p. 226).

La principal distinción entre el EOS y la TAD está en el nivel de desglose que se propone para las praxeologías matemáticas. La noción de sistema de prácticas (operativas, discursivas), ligado a la resolución de un cierto tipo de situaciones-problemas en el que la intervención de un determinado objeto matemático es determinante para su realización, es nuclear en el EOS y se puede asimilar a la noción de praxeología de la TAD (GODINO; FONT; CONTRERAS y WILHELMI, 2006). Sin embargo, mientras que en la TAD la estructura de una praxeología se analiza distinguiendo la cuaterna <tarea, técnica, tecnología, teoría> en el EOS se considera necesario un detalle más explícito de los diversos objetos y procesos que intervienen en la actividad matemática. La noción de configuración de objetos primarios (problemas, lenguajes, conceptos-definición, procedimientos, proposiciones y argumentos) y los procesos de representación, definición, enunciación, argumentación, generalización, entre otros, permiten un nivel de análisis complementario al de la praxeología. En consecuencia, la aplicación de la noción de configuración de objetos y procesos introduce criterios explícitos de idoneidad epistémica referidos a los elementos lingüísticos (representaciones, sus conversiones y tratamientos) y a los respectivos procesos de representación y comunicación (debidamente contemplados en la TSD con las situaciones adidácticas de formulación/comunicación).

Las nociones de tecnología y teoría de la TAD son sustituidas y desglosadas en el EOS por la noción de "configuración de objetos y procesos", lo cual lleva a formular criterios de idoneidad sobre la gestión de los distintos tipos de objetos (conceptos, proposiciones, procedimiento):

- Las definiciones y procedimientos deben ser claros y correctos, y están adaptados al nivel educativo al que se dirigen.

- Se deben presentar los enunciados y procedimientos fundamentales del tema para el nivel educativo dado.

- Se deben proponer situaciones donde los alumnos tengan que generar o negociar definiciones, proposiciones o procedimientos.

En la TAD se reconoce explícitamente el componente procedimental (trabajo de la técnica) como clave en la construcción del conocimiento, lo que queda difuso en la TSD. La TSD, TAD y TID concuerdan en atribuir un papel central a los objetos argumentativos/validativos y a los procesos 
correspondientes de validación/justificación (situaciones de validación, momento tecnológicoteórico).

También se debe prestar atención a las conexiones entre las distintas partes del contenido matemático, y la articulación de los diversos significados parciales de los objetos en estudio (WILHELMI; GODINO y LACASTA, 2007; GODINO; FONT; WILHELMI y LURDUY, 2011). Las matemáticas son un campo de estudio integrado. Esta posición concuerda con el "Principio de interconexión" de la EMR. Los bloques de contenido matemático (numeración y cálculo, álgebra, geometría, ...) no pueden ser tratados como entidades separadas. Las situaciones problemáticas deberían incluir contenidos matemáticos interrelacionados. Además, la resolución de problemas de contexto ricos con frecuencia significa que se requiere aplicar un amplio rango de herramientas y comprensiones matemáticas.

\subsection{Faceta cognitiva}

La dimensión cognitiva es tenida en cuenta en la TAD mediante la noción de "relación personal al objeto" y en la TSD con la distinción entre conocimiento y saber. Sin embargo, el énfasis en la dimensión institucional del conocimiento (TAD) y en las situaciones didácticas (TSD), ha motivado que el foco de atención del análisis didáctico sea el saber matemático (su organización y ecología) y la clase de matemáticas como una institución o comunidad. No obstante, en Chevallard (2009) encontramos una referencia a lo que podemos describir como necesidad de tener en cuenta los conocimientos previos del sujeto para el desarrollo de una actividad o proyecto: "Dado un proyecto de actividad en el que una determinada institución o persona pretende implicarse, ¿cuál es, para esta institución o esta persona, el equipamiento praxeológico que se considera indispensable 0 simplemente útil en la concepción y la realización de ese proyecto" (p. 29).

En el EOS se postula una relación dialéctica entre lo institucional y lo personal, de manera que junto a las configuraciones de objetos y procesos en sentido epistémico (institucional) se introducen las correspondientes configuraciones cognitivas, cuyos elementos constituyentes son los mismos que los de las configuraciones epistémicas. En consecuencia, se formulan criterios de idoneidad cognitiva relacionados con los aprendizajes. Los diversos modos de evaluación deben indicar que los alumnos logran la apropiación de los conocimientos pretendidos (incluyendo distintos niveles de comprensión y competencia): Comprensión conceptual y proposicional; competencia comunicativa y argumentativa; comprensión o competencia procedimental; competencia metacognitiva. 
El principio de nivel de la EMR está relacionado con la faceta cognitiva que propone la TID. Subraya que el aprendizaje de las matemáticas implica que los alumnos pasen por varios niveles de comprensión: desde las soluciones informales relacionadas con el contexto, pasando por la creación de varios niveles de atajos y esquematizaciones, hasta la adquisición de conocimientos sobre cómo se relacionan los conceptos y las estrategias.

\subsection{Faceta afectiva}

La noción de devolución de la TSD se puede interpretar como un componente de la faceta afectiva. Los principios de actividad y realidad de la EMR incorporan aspectos relacionados con la faceta afectiva del aprendizaje. Se aconseja que los alumnos sean tratados como participantes activos en el proceso de aprendizaje, puesto que las matemáticas se aprenden mejor haciendo matemáticas. Se valora de manera explícita el uso de situaciones realistas para los propios estudiantes y tener en cuenta las soluciones informales que desarrollen en su esfuerzo por encontrar solución a dichas situaciones.

Es necesario que los cuatro modelos teóricos considerados adopten o desarrollen modelos explícitos sobre componentes e indicadores de idoneidad relativos al conglomerado de nociones afectivas (intereses, actitudes, emociones), ya que interactúan con la faceta cognitiva y condicionan los aprendizajes. En Beltrán-Pellicer y Godino (2020) se elabora un modelo de análisis del dominio afectivo en educación matemática desde el EOS.

\subsection{Faceta interaccional}

Tanto la TSD (con los tipos de situaciones que se proponen) como la TAD (seis momentos del proceso de estudio) aportan criterios de idoneidad sobre los modos de interacción entre profesor y estudiantes. En el caso de la EMR, con el principio de interactividad se reconoce que el aprendizaje de las matemáticas no es sólo una actividad individual, sino también una actividad social. Por lo tanto, la EMR favorece los debates en toda la clase y el trabajo en grupo, que ofrecen a los estudiantes la oportunidad de compartir sus estrategias e invenciones con los demás. Así mismo, el principio de orientación implica que los profesores deben tener un papel proactivo en el aprendizaje de los alumnos (reinvención guiada de Freudenthal).

Las cuatro teorías con coherentes con los supuestos socio-constructivistas del aprendizaje, un proceso instruccional con alta idoneidad interaccional contempla momentos en los que los estudiantes asumen la responsabilidad del estudio (plantean cuestiones y presentan soluciones; exploran ejemplos y contraejemplos para investigar y conjeturar; usan una variedad de herramientas para razonar, hacer conexiones, resolver problemas y comunicarlos). 
En el caso de la TID se considera que, en los momentos de institucionalización, el profesor deberá hacer una presentación adecuada del tema, reconocer y resolver los conflictos de los alumnos, favorecer los consensos con base al mejor argumento, así como usar diversos recursos retóricos y argumentativos para implicar y captar la atención de los alumnos. Pero estos momentos de institucionalidad pueden tener lugar en cualquier momento del proceso de instrucción cuando se trata del momento de primer encuentro de los estudiantes con un tipo de problema o contenido nuevo, o de recuerdo de contenidos olvidados (GODINO y BURGOS, 2020).

\subsection{Faceta mediacional}

La noción de medio (milieu) es central en la TSD, entendida como el contexto o entorno "antagonista" al que se enfrenta el sujeto para ganar el "juego" del aprendizaje. Es una noción compleja y rica que incluye elementos de diversa naturaleza, conocimientos previos, las acciones del profesor y los medios materiales que se usan para plantear la situación-problema y para abordar y explorar soluciones posibles. En la TAD el milieu no se supone dado al principio con el sistema didáctico (profesor, estudiantes, cuestión), como ocurre en la TSD; el sistema didáctico produce y organiza el medio con el cual, dialécticamente, se genera la respuesta a la cuestión. La faceta mediacional que se introduce en la TID contempla solo los recursos materiales 0 tecnológicos (artefactos) que pueden intervenir en la práctica matemática pretendida, siendo, por tanto, un componente del medio de la TSD.

El uso de recursos tecnológicos no se menciona explícitamente en los seis principios de la EMR; queda implícito en el principio de realidad y también en el uso de modelos en el principio de nivel.

Desde la perspectiva de la RME, Drijvers (2020) considera que la correspondencia con el uso de la tecnología digital no es evidente. La reinvención guiada puede verse desafiada por el carácter rígido de las herramientas, y los fenómenos que forman el punto de partida del aprendizaje de las matemáticas pueden cambiar en un aula rica en tecnología. En cuanto a la fenomenología didáctica, concluye que los fenómenos pueden cambiar en un aula rica en tecnología. El propio entorno digital puede ser un fenómeno significativo para el alumno.

\subsection{Faceta ecológica}

La TAD concede un papel central a la identificación de restricciones y condicionamientos (niveles de codeterminación) en la implementación de las organizaciones didácticas, así como a la articulación entre las distintas praxeologías matemáticas. Se propone evitar el estudio de praxeologías puntuales y aisladas. Estos son componentes de idoneidad que el EOS describe formando parte de la faceta ecológica, la cual queda implícita en la TSD. En el caso de la EMR los 
principios de realidad y de entrelazamiento recogen aspectos de la faceta ecológica; conexión con situaciones de la vida real e integración entre los distintos bloques de contenido.

La TID propone tener en cuenta además de las conexiones entre los distintos contenidos/tópicos/praxeologías matemáticas, y las conexiones interdisciplinares, los siguientes componentes de tipo ecológico:

- La adaptación al currículo;

- Apertura hacia la innovación didáctica;

- Adaptación socio-profesional y cultural; y

- Educación en valores.

\section{Síntesis y observaciones finales}

Una conclusión de este trabajo es que la noción de idoneidad y las diversas facetas, componentes y subcomponentes de un proceso de instrucción, se pueden aplicar para analizar los elementos de diseño y principios educativos de otros marcos teóricos de educación matemática. Aunque la TID se ha desarrollado ligada al EOS, tiene sentido indagar sobre los criterios de idoneidad de los procesos instruccionales diseñados o implementados aplicando la TSD, TAD, EMR u otras teorías. Se abre de este modo, como tema de indagación, la comparación y articulación del componente normativo y axiológico (juicios de valor sobre qué matemáticas enseñar y cómo) de teorías educativas.

En la sección 4 de este artículo hemos mostrado que las cuatro teorías seleccionadas concuerdan en atribuir un papel central al uso de situaciones problemas como razón de ser de la actividad matemática, dando también gran relevancia a la argumentación. El desglose más detallado de los tipos de significados, objetos y procesos que propone el EOS permite formular criterios de idoneidad explícitos para algunas componentes de las facetas epistémica y cognitiva. En la faceta cognitiva se observa más concordancia entre la TID y la EMR que con la TSD y la $T A D$, teorías éstas últimas que centran más su atención en el componente epistémico e interaccional. En la faceta interaccional las cuatro teorías comparten supuestos socioconstructivistas del aprendizaje, atribuyendo un papel importante a los momentos en los que los estudiantes asumen la responsabilidad del estudio. Se han observado diferencias importantes en cómo conciben las teorías el medio (milieu) y el contexto ecológico en que tiene lugar el proceso instruccional; en consecuencia, resulta difícil comparar los respectivos criterios de idoneidad correspondientes a estas facetas. 
La ingeniería didáctica, en el sentido usado en la didáctica francesa, tiene unas características propias que la distinguen de las restantes investigaciones de diseño educativo. El hecho que se use como teoría base la TSD, la TAD u otras, hace que el objetivo principal de las experiencias que se diseñan, implementan y evalúan esté orientado hacia la investigación de tipo fundamental, esto es, la descripción y explicación de fenómenos didácticos (GASCÓN y NICOLÁS, 2021). Dado que lo propio del trabajo del ingeniero es la intervención en una parcela de la realidad para su mejora, siguiendo unas pautas o normas previamente establecidas, podríamos decir que la "ingeniería didáctica de investigación" no es realmente una ingeniería, sino una metodología de investigación orientada a la comprensión de los procesos de difusión del conocimiento matemático. Este no es el caso de las investigaciones que se realizan en el marco de la EMR que, aunque parten de unos principios epistemológicos, cognitivos y educativos para sus diseños curriculares e instruccionales tienen como objetivo principal la elaboración de modelos teóricos locales y recursos que optimicen la enseñanza y aprendizaje de contenidos matemáticos específicos. En el caso de EOS se han elaborado principios y herramientas metodológicas para abordar cuestiones descriptivas y explicativas de carácter fundamental, las cuales pueden ser de índole epistemológica, ontológica, semiótica y cognitiva, y también cuestiones predictivas, esto es, de diseño 0 ingeniería didáctica de desarrollo (GODINO et al., 2014). La noción de idoneidad didáctica, sus facetas, componentes, subcomponentes y criterios permite establecer un puente entre las cuestiones de diseño y la investigación-acción realizada por los propios docentes.

El modelo del "Cuadrante de Pasteur" propuesto por Stokes (1997) ayuda a comprender las relaciones entre la investigación básica o fundamental, la investigación aplicada y la práctica de la enseñanza. Ayuda también a reconocer el papel de la noción de idoneidad como nexo entre la investigación científico-tecnológica y la práctica reflexiva. Stokes (1997) clasifica las investigaciones según dos ejes: si persiguen o no la búsqueda de la comprensión fundamental; si están inspiradas o no por consideraciones de uso, obteniendo, de este modo un modelo con cuatro cuadrantes. En la Figura 3 hacemos una interpretación y adaptación de este modelo para el caso de la educación matemática, y en particular para las teorías mencionadas en este trabajo.

En el cuadrante I (ejemplificado por la investigación de Louis Pasteur según Stokes) incluimos las investigaciones orientadas al diseño (investigación básica aplicada) en la que incluimos la EMR. En el cuadrante II se tienen las investigaciones de tipo fundamental (básica pura) en el que incluimos la TSD y la TAD, entre cuyos componentes está la "ingeniería didáctica de investigación" (ID-I). La ingeniería didáctica de desarrollo (ID-D) se relaciona también con el cuadrante 1. El cuadrante III no está vacío; Stokes incluye aquí la investigación que explora 
sistemáticamente fenómenos particulares, sin tener en cuenta los objetivos explicativos generales ni el uso aplicado que se dará a los resultados. En el caso de la investigación educativa se puede representar el cuadrante III con la indagación de tipo naturalista en sus diferentes versiones (etnografías, estudios de casos, biografías,...). El cuadrante IV puede estar representado por la práctica reflexiva (investigación-acción en sus diferentes versiones); el foco de atención es la mejora de la práctica, bien de manera colaborativa o individual.

En la Figura 3 hemos situado las investigaciones basadas en el EOS entre los cuatro cuadrantes, al considerar que aporta herramientas y principios para abordar investigaciones de carácter fundamental y aplicado, y también para la práctica reflexiva mediante la TID. De la misma manera que el trabajo del ingeniero se caracteriza por el seguimiento de las normas técnicas establecidas para la ejecución de sus trabajos, lo que le garantiza la idoneidad de los mismos, el trabajo del profesor debería progresar en esa misma dirección. Es cierto que hay una diferencia esencial entre el campo de las ciencias naturales, en las que las leyes que rigen los fenómenos que estudian tienen una cierta generalidad, y las ciencias sociales, caracterizadas por la dependencia del contexto y el carácter local de sus resultados. Sin embargo, ello no debería llevar a la conclusión de que no existen criterios que guíen el trabajo del profesor.

Figura 3. Investigación fundamental y aplicada en educación matemática

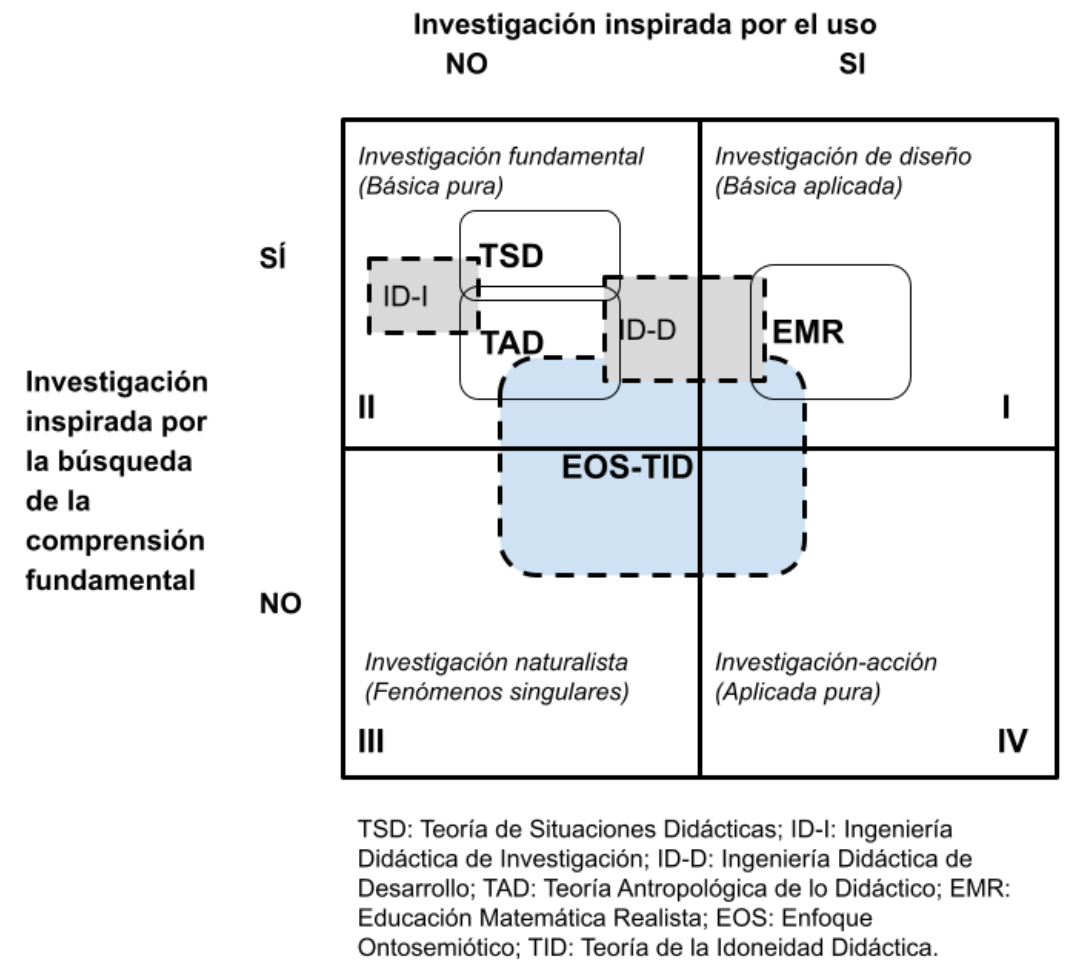

Fuente: Adaptado de Stokes (1997, p. 73) 
Aunque se han publicado un número relativamente alto de trabajos con aplicaciones de la TID (MALET; GIACOMONE y REPETTO, 2021) reconocemos la necesidad de profundizar en su desarrollo, refinando los criterios e indicadores generales de idoneidad y desarrollando criterios específicos para diferentes contenidos. En cierta manera, los distintos marcos teóricos usados en educación matemática asumen de manera implícita criterios de idoneidad propios, como hemos tratado de mostrar en este trabajo para la TSD, TAD y EMR. La clarificación y comparación de estos criterios de idoneidad de diferentes marcos teóricos y su articulación en un sistema coherente supone un programa de investigación que apenas hemos sugerido en este artículo.

\section{Reconocimiento}

Trabajo realizado en el marco del Proyecto PID2019-105601GB-I00/AEI/ 10.13039/501100011033 y en el seno del Grupo FQM-126 del PAI (Junta de Andalucía, España).

\section{Referencias:}

ARTIGUE, Michèle. Ingénierie didactique. Recherches en Didactique des Mathématiques, v. 9 , n. 3, p. 281-308, 1989.

ARTIGUE, Michèle. Didactical design in mathematics education. In C. Winsløw (Ed.), Nordic research in mathematics education. Proceedings from NORMA08 (pp. 5-16). Sense Publishers, 2009.

ARTIGUE, Michèle. L'ingénierie didactique: un essai de synthèse. In C. Margolinas, M. AbboudBlanchard, L. Bueno-Ravel, N. Douek, A. Fluckiger, P. Gibel, F. Vandebrouck y F. Wozniak (Eds.), En amont et en aval des ingénieries didactiques (pp. 225-237). Grenoble: La Pensée Sauvage, 2011.

ARTIGUE, Michèle. Perspectives on Design Research: The Case of Didactical Engineering. In Angelika BIKNER-AHSBAHS; Christine KNIPPING; Norma PRESMEG (Eds.), Approaches to qualitative research in mathematics education. Examples of methodology and methods. (pp. 467-496). Springer, 2015.

BAKKER, Arthur; VAN EERDE, Dolly. An introduction to design-based research with an example from statistics education. In Angelika BIKNER-AHSBAHS; Christine KNIPPING; Norma PRESMEG (Eds.), Approaches to qualitative research in mathematics education. Examples of methodology and methods. (pp. 429-466). Springer, 2015

BELTRÁN-PELLICER, Pablo; GODINO, Juan D. An onto-semiotic approach to the analysis of the affective domain in mathematics education. Cambridge Journal of Education, v. 50, n. 1, p. 120, 2020. 
BREDA, Adriana; FONT, Vicenç; PINO-FAN, Luis. Criterios valorativos y normativos en la Didáctica de las Matemáticas: el caso del constructo idoneidad didáctica. Bolema, v. 32, n. 60, p. $255-278,2018$.

BROWN, Ann L. Design experiments: Theoretical and methodological challenges in creating complex interventions in classroom settings. The Journal of the Learning Sciences, v. 2, n. 2, p. 141-178, 1992.

BROUSSEAU, Guy. Fondements et méthodes de la didactique des mathématiques. Recherches en Didactique des Mathématiques, v. 7, n. 2, p. 33-115, 1986.

BROUSSEAU, Guy. Theory of didactical situations in mathematics. Dordrecht, The Netherlands: Kluwer, 1997.

BUNGE, Mario. Las ciencias sociales en discusión: una perspectiva filosófica. Buenos Aires, Editorial Sudamericana, 1999.

CHEVALLARD, Yves. Concepts fondamentaux de la didactique: perspectives apportées par une approche anthropologique. Recherches en Didactique des Mathématiques, v. 12, n. 1, p. 73112, 1992.

CHEVALLARD, Yves. Familière et problématique, la figure du professeur. Recherches en Didactique des Mathématiques, v. 17, n. 3, p. 17-54, 1997.

CHEVALLARD, Yves. L'analyse des pratiques enseignantes en théorie anthropologique du didactique. Recherches en Didactique des Mathématiques, v. 19, n. 2, p. 221-266, 1999.

CHEVALLARD, Yves. La notion d'ingénierie didactique, un concept à refonder. Questionnement et éléments de réponse à partir de la TAD. 15e École d'Été de Didactique des Mathématiques. Clermont-Ferrand, 2009. On line, http://yves.chevallard.free.fr/.

CHEVALLARD, Yves.; BOSCH, Mariana y GASCÓN, Josep. Estudiar matemáticas. El eslabón perdido entre la enseñanza y el aprendizaje. Barcelona: ICE-Horsori, 1997.

COBB, Paul; GRAVEMEIJER, Koeno. Experimenting to support and understand learning processes. In Anthony E. KELLY; Richard A. LESH; John Y. BAEK (Eds.), Handbook of design research methods in education. Innovations in Science, Technology,Engineering and Mathematics Learning and Teaching (pp. 68-95). Mahwah, NJ: Lawrence Erlbaum, 2008.

DRIJVERS, Paul. Digital tools in Dutch mathematics education: a dialectic relationship. In M. VAN DEN HEUVEL-PANHUIZEN (Ed.), National reflections on the Netherlands didactics of mathematics, iCME-13 Monographs, (pp. 177-195). Springer, 2020.

FREUDENTHAL, Hans. Mathematics as an educational task. Reidel Publishing, Dordrecht, 1973.

FREUDENTHAL, Hans. Didactical phenomenology of mathematical structures. Reidel Publishing, Dordrecht, 1983.

FREUDENTHAL, H. Revisiting mathematics education. China lectures. Kluwer, Dordrecht, 1991. 
GASCÓN, Josep. Evolución de la didáctica de las matemáticas como disciplina científica. Recherches en Didactique des Mathématiques, v. 18/1 n. 52, p. 7-33, 1998.

GASCÓN, Josep; NICOLÁS, Pedro. Incidencia de los paradigmas didácticos sobre la investigación didáctica y la práctica docente. Educación Matemática, v. 33, n. 1, p.7-40, 2021.

GODINO, Juan D. Un enfoque ontológico y semiótico de la cognición matemática. Recherches en Didactiques des Mathematiques, v. 22, n. (2/3), p. 237-284, 2002.

GODINO, Juan D. Indicadores de la idoneidad didáctica de procesos de enseñanza y aprendizaje de las matemáticas. Cuadernos de Investigación y Formación en Educación Matemática, v. 11, p. 111-132, 2013.

GODINO, Juan D.; BATANERO, Carmen; BURGOS, María y GEA, María M. Una perspectiva ontosemiótica de los problemas y métodos de investigación en educación matemática. Revemop, 3, e202107, p. 1-30, 2021. https://periodicos.ufop.br/revemop/article/view/4848/3773

GODINO, Juan D.; BATANERO, Carmen; FONT, Vicenç. The onto-semiotic approach to research in mathematics education. ZDM. The International Journal on Mathematics Education, v. 39, n. 1-2, p. 127-135, 2007.

GODINO, Juan D.; BATANERO, Carmen; FONT, Vicenç. The onto-semiotic approach: implications for the prescriptive character of didactics. For the Learning of Mathematics, v. 39, n. 1, p. 37- 42, 2019.

GODINO, Juan D.; BURGOS, María. ¿Cómo enseñar las matemáticas y las ciencias experimentales? Resolviendo el dilema de la indagación y transmisión. Paradigma, v. XLI, p. 80 106, 2020.

GODINO, Juan D.; FONT, Vicenç; CONTRERAS, Angel; WILHELMI, Miguel. R. Una visión de la didáctica francesa desde el enfoque ontosemiótico de la cognición e instrucción matemática. Revista Latinoamericana de Investigación en Matemática Educativa, v. 9, n. 1, p. 117-150, 2006.

GODINO, Juan D.; FONT, Vicenç; WILHELMI, Miguel R.; LURDUY, Orlando. Why is the learning of elementary arithmetic concepts difficult? Semiotic tools for understanding the nature of mathematical objects. Educational Studies in Mathematics, v. 77, n. 2, p. 247-265, 2011.

GODINO, Juan D.; RIVAS, Hernán; ARTEAGA, Pedro; LASA, Aitzol; WILHELMI, M. R. Ingeniería didáctica basada en el enfoque ontológico - semiótico del conocimiento y la instrucción matemáticos, Recherches en Didactique des Mathématiques, v. 34, n. 2/3, p. 167-200, 2014.

GRAVEMEIJER, Koeno. A Socio-constructivist elaboration of realistic mathematics education. In M. VAN DEN HEUVEL-PANHUIZEN (Ed.), National reflections on the Netherlands didactics of mathematics, ICME-13 Monographs, (pp. 217-233). Springer, 2020.

KELLY, Anthony E.; LESH, Richard A.; BAEK, John Y. (Eds.). Handbook of design research in methods in education. Innovations in science, technology, engineering, and mathematics learning and teaching. New York, NY: Routledge, 2008.

LACEY, Hugh. Is science value free? Values and scientific understanding. Routledge, 1999. 
MALET, Omar; GIACOMONE, Belén; REPETTO, Ana M. La Idoneidad didáctica como herramienta metodológica: desarrollo y contextos de uso. Revemop, 3, e202110, 1-23, 2021. https://doi.org/10.33532/revemop.e202110

MORIN, Edgar. Introducción al pensamiento complejo. Barcelona: Gedisa, 1994.

REIGELUTH, Charles M. ¿En qué consiste una teoría de diseño educativo y cómo se está transformando? In Charles M. REIGELUTH (Ed.), Diseño de la instrucción. Teorías y modelos. Un Nuevo paradigma de la teoría de la instrucción (pp. 15-40). Madrid: Santillana, 2000.

RUGINA, Anghel. N. The problem of values and value-judgments in science and a positive solution: Max Weber and Ludwig Wittgenstein revisited. International Journal of Social Economics, v. 25, n. 5, p. 805-854, 1998. https://doi.org/10.1108/EUM0000000004522.

SIERPINSKA, Anna; LERMAN, Stephen. Epistemologies of mathematics and of mathematics education. In A. J. BISHOP et al. (eds.), International Handbook of Mathematics Education (pp. 827-876). Dordrecht, The Netherlands: Kluwer, 1996.

STOKES, Donald. Paster's quadrant. Basic science and technological innovation. Washington, D.C: Brookings Institution Press, 1997.

TREFFERS, Adrian. Three dimensions. A model of goal and theory description in mathematics instruction - the Wiskobas project. D. Reidel Publishing, Dordrecht, 1987.

VAN DEN HEUVEL-PANHUIZEN, Marja. The didactical use of models in realistic mathematics education: an example from a longitudinal trajectory on percentage. Educational Studies in Mathematics, v. 54, n. 1, p. 9-35, 2003.

VAN DEN HEUVEL-PANHUIZEN, Marja; DRIJVERS, P. Realistic mathematics education. In Stephen Lerman (Ed.), Encyclopedia of Mathematics Education, 2014. https://doi.org/10.1007/978-94-007-4978-8.

WILHELMI, Miguel R.; GODINO, Juan D.; LACASTA, Eduardo. Configuraciones epistémicas asociadas a la noción de igualdad de números reales. Recherches en Didactique des Mathematiques, v. 27, n. 1, p. $77-120,2007$.

VYGOTSKY, Lev S. El desarrollo de los procesos psicológicos superiores. Barcelona: CríticaGrijalbo, 1934. 